\title{
Application of High-Quality Nursing Intervention Based on Humanistic Care Combined with the Project Teaching Method in Patients with Acute Leukemia Undergoing Chemotherapy
}

\author{
Zhiyan Wang $(\mathbb{D}$ \\ Department of Hematology Oncology, Tianjin Fifth Central Hospital, Tianjin 300450, China \\ Correspondence should be addressed to Zhiyan Wang; wangzhiyan@tjwzx.org.cn
}

Received 8 December 2021; Revised 23 December 2021; Accepted 27 December 2021; Published 9 February 2022

Academic Editor: Kalidoss Rajakani

Copyright (c) 2022 Zhiyan Wang. This is an open access article distributed under the Creative Commons Attribution License, which permits unrestricted use, distribution, and reproduction in any medium, provided the original work is properly cited.

\begin{abstract}
Chemotherapy, the main treatment method of AL, can produce varying degrees of toxic and side effects on patients while killing tumor cells, resulting in decreased immune function of patients. We aim to explore the application effect of high-quality nursing intervention based on humanistic care combined with the project teaching method on patients with acute leukemia (AL) undergoing chemotherapy and its effect on their psychological state and satisfaction. 114 AL patients undergoing chemotherapy in the Department of Hematology of our hospital from July 2018 to July 2020 were chosen as the research objects and equally randomized into the experimental group (EG) and control group (CG). CG received routine nursing during chemotherapy, while EG received high-quality nursing intervention based on humanistic care combined with the project teaching method. Hospital Anxiety and Depression Scale (HAD) was adopted to evaluate the psychological state of patients in both groups after intervention, and the self-made nursing satisfaction questionnaire in our hospital was applied to evaluate the clinical nursing satisfaction. The HAD-A and HAD-D scores in the CG $(P<0.001)$ were significantly higher than the HAD-A and HAD-D scores in the EG after intervention. The scores of various coping styles in the EG after intervention were better than those in the CG $(P<0.05)$. Compared with the CG, the total nursing satisfaction after intervention was remarkably higher in the EG $(P<0.001)$, while the total incidence of adverse reactions during intervention was notably lower $(P<0.05)$. High-quality nursing intervention based on humanistic care combined with the project teaching method for AL patients undergoing chemotherapy can effectively relieve negative emotions, improve clinical nursing satisfaction, and reduce adverse reactions during chemotherapy.
\end{abstract}

\section{Introduction}

Acute leukemia (AL), a common malignant tumor of hematopoietic stem cells, often presents with symptoms such as bleeding, anemia, infiltration, and infection [1]. We can clinically split AL into two parts according to the cell types involved: acute lymphoblastic leukemia (ALL) and acute myeloid leukemia (AML). The primary symptoms of AL included persistent high fever or repeated infection with enlargement of the spleen, liver, and lymph nodes to varying degrees, resulting in only a few months of survival time in most patients without treatment. Chemotherapy is a common treatment method for AL to inhibit tumor growth in patients [2]. A clinical study [3] has shown that although the condition in $65 \%$ of patients can be effectively alleviated after the first chemotherapy and $20 \%$ of the patients can live for more than five years, there are also a high recurrence rate and incidence of adverse reactions, affecting the treatment compliance and quality of life of patients and leading to depression. Since negative emotions can make the psychological state of patients more vulnerable, strengthening the nursing intervention for such patients during chemotherapy is convincing to reduce negative emotions and increase the therapeutic effect [4-6]. High-quality nursing intervention based on humanistic care pays attention to the changes of patients' physical and mental feelings, enables them to feel the care from medical staff through various nursing means, and improves their nursing satisfaction, which has been confirmed in patients with radical mastectomy [7]. The project teaching method is centered on 
team learning, takes course projects as the main line, takes task goals as the mission, and emphasizes the ability, quality, and professional knowledge of medical staff. It can effectively cultivate nurses' sense of teamwork and innovation and improve nursing satisfaction, which has been proved in advanced cancer [8]. Modern society attaches great importance to teamwork and innovation and requires all team members to cooperate actively with each other to form the highest synergy effect. Some scholars $[9,10]$ pointed out in their studies that many students expressed that the project teaching method, especially the thinking mode of highlighting team characteristics by naming clinical departments, reduced the strangeness and fear of clinical practice and made them quickly adapt to clinical nursing. Be founded on this, this study proposes to investigate the application effect of high-quality nursing intervention based on humanistic care combined with the project teaching method on AL patients undergoing chemotherapy and its effect on their psychological state and satisfaction.

\section{Materials and Methods}

2.1. Data Collection. In this prospective randomized controlled clinical study, we selected $114 \mathrm{AL}$ patients undergoing chemotherapy in the Department of Hematology of our hospital from July 2018 to July 2020. The inclusion criteria were as follows: (1) the patients satisfied the powerful diagnostic criteria of AL in Leukemia Diagnostics [11], confirmed by bone marrow puncture; (2) the patients were no more than 65 years old; (3) the anticipated survival time of the patients was more than 3 months; (4) the patients did not receive antitumor drugs, biological immunotherapy, or other drug treatments before admission; (5) the patients received national uniform regimens of chemotherapy, with methotrexate as the main chemotherapy drug; and (6) the study conformed with the hospital ethics institutional mechanisms, and the patients knew and approved informed consent. The exclusion criteria were as follows: (1) the patients with allergy to chemotherapy drugs; (2) the patients complicated with liver, kidney, cardiopulmonary, and brain dysfunction; (3) the patients with mental and other cognitive dysfunctions; and (4) the patients complicated with other malignant tumors.

2.2. Methods. The patients in the CG received routine nursing during treatment. After admission, they were informed of the hospital conditions (hospital environment and hospital rules and regulations), the specific process, adverse reactions, and precautions of chemotherapy in detail. The health education paths for patients with chemotherapy were developed by the medical staff of the department, including disease cognition, medication management, diet management, and psychological nursing, and education was carried out through distribution of health promotion manuals and centralized or individual oral education. The patients also received monitoring of the vital signs, basic drug nursing and life nursing, and appropriate treatment of complications [12-14].
The patients in the EG received high-quality nursing intervention based on humanistic care combined with the project teaching method. The specific steps of high-quality nursing based on humanistic care were as follows. (1) The actual needs of patients were analyzed to master their specific psychological status, mental state, and their own cognition of the disease. Medical staff communicated with patients (1 time/day and $15 \mathrm{~min}$ each time) to understand their real thoughts and needs and encourage them to bravely express their feelings. The staff also provided timely nursing for patients and their families, improved their quality of life during chemotherapy, reduced the side effects of chemotherapy, respected their original lifestyles as much as possible, and arranged them to do what they could do according to their condition. The staff should have enough patience to understand the patients' emotions. (2) Targeted programs should be adopted according to different psychological emotions of patients to timely solve their psychological problems, eliminate adverse emotions, and reduce their fear of chemotherapy. Meanwhile, the staff should also carry out death education for patients to eliminate their anxiety and fear when facing death, which could also help their families to ease their grief. (3) The pain of patients during chemotherapy was minimized, and analgesic treatment should be carried out for patients who could not tolerate the pain. If the pain of patients was not alleviated, other analgesic schemes could be adopted, or other analgesic methods could be applied jointly, such as slow breathing and distraction. Necessary care and concern were given to patients. In addition to drug treatment, appropriate comfort should be provided when the pain occurred during chemotherapy, which reflected humanistic care. Besides, targeted nursing measures should be taken to reduce the physical pain of patients and soothe their bad emotions. (4) The family members were instructed to make food with high vitamin and protein contents for patients. Food should be easy to digest and not be too greasy, with more fresh fruits and vegetables. The wards were kept quiet, clean, and comfortable with appropriate temperature and humidity. The patients were advised to keep adequate sleep every day and walk properly in the wards if circumstances permitted to relieve the gastrointestinal discomfort caused by chemotherapy. (5) The patients with adverse reactions after chemotherapy should be actively intervened by strengthening perianal, perineal, and oral nursing. For example, the patients were advised to brush their teeth before and after eating and before sleeping, rinse the mouth with salt water, and maintain normal defecation to prevent constipation. Before chemotherapy, they orally took omeprazole to reduce the damage to gastric mucosa. The number of vomiting and the nature of vomit were carefully recorded, and the changes in skin color were observed [15-17].

Project teaching method was implemented as follows. (1) According to the clinical situation of typical cases, clinical teaching projects were formulated. The nurse with more than 6 years of experience in the department acted as the team leader, and other team members were nursing staff in the department to dynamically observe the information of patients in each ward. In this study, taking AL patients who 
underwent chemotherapy as an example, since the project was to develop the nursing plans for such patients, the team leader should consider the following aspects in the selection of cases. (a) The typicality of the disease: since the focus of the training was to cultivate the clear clinical thinking ability of the nursing staff, the cases should not be too complex. (b) The stability of the disease: the treatment pain of the patients should not be increased due to the project teaching. (c) Oral informed consent of patients and their families: the project teaching method started from the project selection, and the team leader should actively invite the team members to participate in the teaching project selection. (2) Teaching projects: they included teaching content, exploring projects, discussing problems, teaching operation, practical operation of team members, primary nurses, and expansion of new knowledge and new technology. (3) Division of teaching projects: guided by the team leader, each member of the group played a corresponding role and completed the task in the teaching projects. Every two nurses could undertake a task for supplement and comparison and carry out training on nurse-patient communication skills and humanistic care. (4) The nursing staff followed the doctors to make ward rounds and collect various clinical information of patients. If there were any doubts, they should timely consult the team leader and formulate nursing measures. The nursing measures should be matched with the problems one by one, which should be symptomatic, effective, economical, and operable, until the nursing plans were improved [18].

2.3. Evaluation Indexes. The anxiety and depression of patients before and after intervention were evaluated by referring to the Hospital Anxiety and Depression Scale (HAD) score [19]. The scale was divided into the HAD-A (for anxiety) score and HAD-D (for depression) score, with a sum of 15 points for each. The higher the score is, the more serious the negative emotions are.

The medical coping mode questionnaire (MCMQ) [20] was adopted to evaluate the coping styles of patients before and after intervention, including 20 items in three dimensions of avoidance, surrender, and facing and each scoring 4 points. The higher the score, the stronger the corresponding dimension.

The self-made nursing satisfaction questionnaire in our hospital was used to evaluate the satisfaction of both groups with clinical nursing. The full score of the questionnaire was 100 points, and a score of $80-100$ points showed the patients were fully satisfied, with $60-79$ as satisfied and $0-59$ as dissatisfied. Total percentage of satisfaction $=($ fully satisfied cases + satisfied cases)/sum of cases $\times 100 \%$.

2.4. Statistical Methods. The whole collected data were statistically analyzed and operated by SPSS 21.0 software and graphed by GraphPad Prism 7 (GraphPad Software, San Diego, USA). Enumeration data were tested by $X^{2}$ and represented as $N(\%)$, while measurement data were tested by the $t$-test and expressed as $(\bar{x} \pm s)$. The differences were statistically significant at $P<0.05$.

\section{Results}

3.1. Comparison of Baseline Data. There are no obvious dissimilarities in sex ratio, average course of the disease, severity of the disease, disease types, marital status, residence, and education between the two groups $(P>0.05$; Table 1$)$.

\subsection{Comparison of HAD Scores before and after Intervention.} The HAD-A and HAD-D scores in the EG were obviously different before and after intervention $(P<0.001)$, and the scores in the EG after intervention were significantly lower than the CG $(P<0.001$; Figure 1$)$.

3.3. Comparison of Coping Style Scores before and after Intervention. The scores of various coping styles in the EG after intervention were better than those in the CG $(P<0.05$; Table 2).

3.4. Comparison of Clinical Satisfaction. Compared with the CG, the total satisfaction after intervention was remarkably higher in the EG $(P<0.001$; Table 3$)$.

3.5. Comparison of the Occurrence of Adverse Reactions during Chemotherapy. The entire incidence of unfavorable reactions in the EG during intervention was remarkably lower than in the CG $(P<0.05$; Table 4$)$.

\section{Discussion}

AL will increase the psychological burden of patients and inevitably bring psychological and physiological stress responses to them. The pain caused by chemotherapy will increase the degree of stress response and affect the treatment compliance of patients [21]. AL patients should receive chemotherapy as early as possible to inhibit tumor growth, delay disease progression, and improve survival rate. However, some scholars [22] have found that chemotherapy drugs have both positive and negative effects, which can induce apoptosis of tumor cells, increase the release of 5-hydroxytryptamine in enterochromaffin cells, and activate 5-hydroxytryptamine receptors in the central nervous system, leading to a series of gastrointestinal adverse reactions. Studies $[23,24]$ have confirmed the high 5-hydroxytryptamine level in blood of patients with mental depression, which demonstrates that reducing the 5-hydroxytryptamine level can be achieved by improving patients' adverse emotions, thereby reducing gastrointestinal adverse reactions. Therefore, effective nursing intervention measures can increase the adverse emotions of AL patients, decrease the toxic and side effects of chemotherapy, and improve the therapeutic effect.

In this study, high-quality nursing intervention based on humanistic care combined with the project teaching method was implemented for patients in the EG. After intervention, the anxiety and depression of patients were remarkably improved because the high-quality nursing intervention based on humanistic care can provide high-quality clinical nursing support for AL patients undergoing chemotherapy, 
TABLE 1: Comparison of baseline data $(n(\%))$.

\begin{tabular}{|c|c|c|c|c|}
\hline Items & $\mathrm{EG}(n=57)$ & CG $(n=57)$ & $X^{2} / t$ & $P$ \\
\hline Gender & & & 0.141 & 0.708 \\
\hline Male & $31(54.39 \%)$ & $29(50.88 \%)$ & & \\
\hline Female & $26(45.61 \%)$ & $28(49.12 \%)$ & & \\
\hline Average age $(\bar{x} \pm s$, years old $)$ & $32.67 \pm 2.76$ & $32.83 \pm 2.69$ & 0.313 & 0.755 \\
\hline BMI $\left(\mathrm{kg} / \mathrm{m}^{2}\right)$ & $21.46 \pm 1.64$ & $21.48 \pm 1.59$ & 0.066 & 0.947 \\
\hline Average course of the disease (weeks) & $3.46 \pm 0.35$ & $3.51 \pm 0.28$ & 0.842 & 0.402 \\
\hline \multicolumn{5}{|l|}{ Severity of the disease } \\
\hline High risk & $31(54.39 \%)$ & $29(50.88 \%)$ & 0.141 & 0.708 \\
\hline Medium risk & $22(38.60 \%)$ & $23(40.35 \%)$ & 0.037 & 0.848 \\
\hline Low risk & $4(7.02 \%)$ & $5(8.77 \%)$ & 0.121 & 0.728 \\
\hline Disease types & & & 0.035 & 0.851 \\
\hline AML & $27(47.37 \%)$ & $28(49.12 \%)$ & & \\
\hline ALL & $30(52.63 \%)$ & $29(50.88 \%)$ & & \\
\hline \multicolumn{5}{|l|}{ Marital status } \\
\hline Unmarried & $9(15.79 \%)$ & $11(19.30 \%)$ & 0.243 & 0.622 \\
\hline Married & $43(75.44 \%)$ & $42(73.68 \%)$ & 0.046 & 0.830 \\
\hline Divorced & $5(8.77 \%)$ & $4(7.02 \%)$ & 0.121 & 0.728 \\
\hline Residence & & & 0.141 & 0.708 \\
\hline Urban area & $26(45.61 \%)$ & $28(49.12 \%)$ & & \\
\hline Rural area & $31(54.39 \%)$ & $29(50.88 \%)$ & & \\
\hline \multicolumn{5}{|l|}{ Education } \\
\hline College and above & $17(29.82 \%)$ & $19(33.33 \%)$ & 0.162 & 0.687 \\
\hline High school & $33(57.89 \%)$ & $29(50.88 \%)$ & 0.566 & 0.452 \\
\hline Middle school and below & $7(12.28 \%)$ & $9(15.79 \%)$ & 0.291 & 0.590 \\
\hline
\end{tabular}

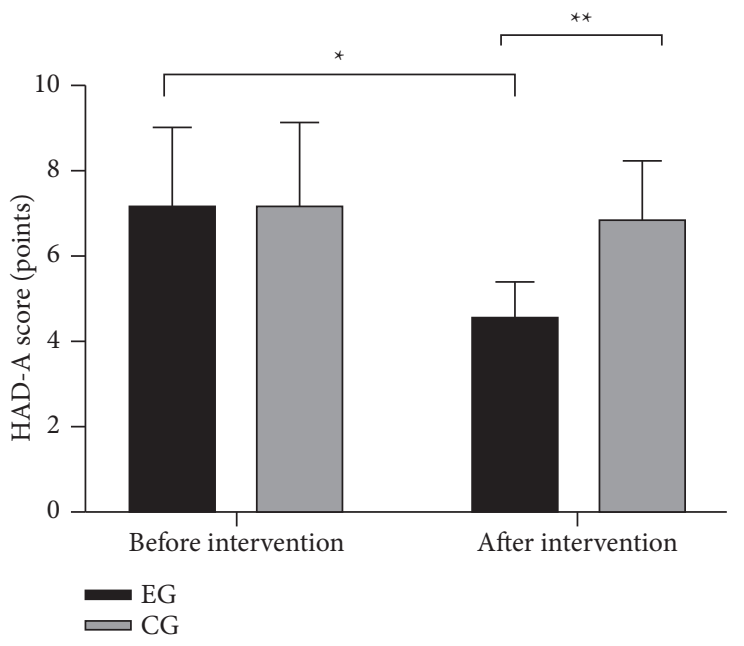

(a)

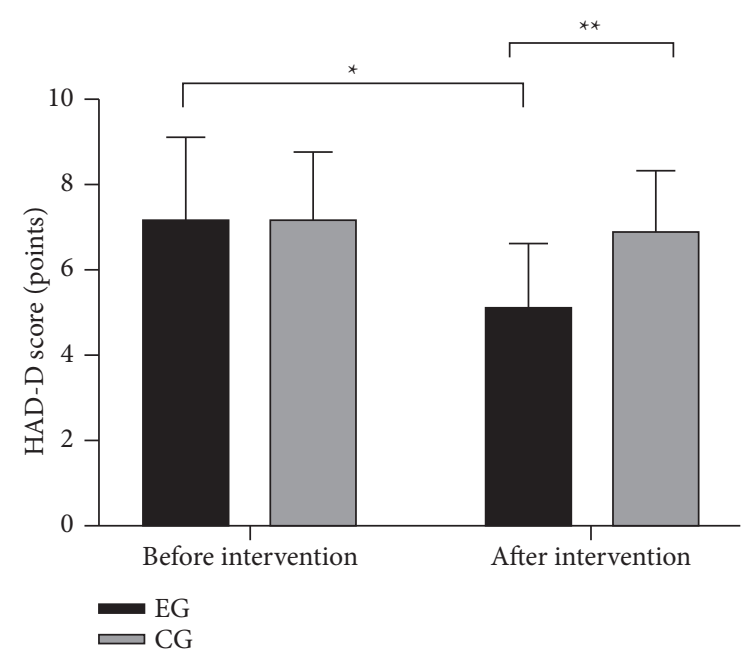

(b)

FiguRE 1: Comparison of HAD scores before and after intervention $(\bar{x} \pm s)$. (a) Comparison of HAD-A scores before and after intervention. The abscissa described before and after intervention, and the ordinate described the HAD-A score (points). The HAD-A scores of the EG before and after treatment were $7.21 \pm 1.83$ and $4.62 \pm 0.81$, while those of the CG were $7.24 \pm 1.92$ and $6.94 \pm 1.32 .{ }^{*}$ A notable difference in the HAD-A scores of the EG before and after intervention $(t=9.771, P<0.001)$; ${ }^{* *}$ a significant dissimilarity in the HAD-A scores after intervention $(t=7.827, P<0.001)$. (b) Comparison of HAD-D scores before and after intervention. The abscissa described before and after intervention, and the ordinate described the HAD-D score (points). The HAD-D scores of the EG were $7.28 \pm 1.83$ before treatment and $5.13 \pm 1.45$ after treatment, while those of the CG were $7.23 \pm 1.54$ and $6.92 \pm 1.36{ }^{*}$ A notable difference in the HAD-D scores of the EG before and after intervention $(t=6.952, P<0.001)$; ${ }^{* *}$ a notable difference in the HAD-D scores after intervention $(t=6.798, P<0.001)$.

strictly implement the core idea of patient-centered nursing, provide emotional support for them, improve their psychological, spiritual, and physiological comfort, and enhance the nursing quality. In addition, in terms of coping styles, the symptomatic nursing for patients enabled them to face death directly and improve the courage and confidence of treatment. Therefore, the scores of various coping styles in the EG after intervention were better than those in the CG $(P<0.05)$, which was consistent with the study of Yang et al. [25]. The project teaching method simulates the clinical nursing team 
TABLE 2: Comparison of coping style scores before and after intervention ( $\bar{x} \pm s$, points).

\begin{tabular}{|c|c|c|c|c|c|c|}
\hline \multirow{2}{*}{ Group } & \multicolumn{2}{|c|}{ Facing } & \multicolumn{2}{|c|}{ Surrender } & \multicolumn{2}{|c|}{ Avoidance } \\
\hline & Before intervention & After intervention & Before intervention & After intervention & Before intervention & After intervention \\
\hline $\mathrm{EG}(n=57)$ & $14.28 \pm 3.18$ & $22.16 \pm 3.14$ & $21.16 \pm 2.18$ & $14.28 \pm 1.87$ & $13.28 \pm 2.23$ & $7.59 \pm 1.58$ \\
\hline CG $(n=57)$ & $14.31 \pm 3.09$ & $16.25 \pm 2.78$ & $21.19 \pm 2.11$ & $17.26 \pm 2.18$ & $13.32 \pm 2.35$ & $11.02 \pm 1.47$ \\
\hline$t$ & 0.051 & 10.639 & 0.075 & 7.833 & 0.093 & 11.100 \\
\hline$P$ & 0.959 & $<0.001$ & 0.941 & $<0.001$ & 0.926 & $<0.001$ \\
\hline
\end{tabular}

TABLE 3: Comparison of clinical satisfaction ( $n(\%))$.

\begin{tabular}{|c|c|c|c|c|c|}
\hline Group & $n$ & Fully satisfied & Satisfied & Dissatisfied & Total satisfaction \\
\hline EG & 57 & $19(33.33 \%)$ & $36(63.16 \%)$ & $2(3.51 \%)$ & $96.49 \%(55 / 57)$ \\
\hline CG & 57 & $20(35.09 \%)$ & $28(49.12 \%)$ & $9(15.79 \%)$ & $84.21 \%(48 / 57)$ \\
\hline$X^{2}$ & & & & & 4.930 \\
\hline$P$ & & & & & $<0.05$ \\
\hline
\end{tabular}

TABLE 4: Comparison of the occurrence of unfavorable reactions during chemotherapy ( $n(\%))$.

\begin{tabular}{|c|c|c|c|c|c|c|}
\hline Group & $n$ & Myelosuppression & Gastrointestinal discomfort & Cardiotoxicity & Respiratory tract infection & Total incidence \\
\hline EG & 57 & $1(1.75)$ & $2(3.51)$ & $0(0.00)$ & $1(1.75)$ & $7.02 \%(4 / 57)$ \\
\hline CG & 57 & $3(5.26)$ & $4(7.02)$ & $3(5.26)$ & $3(5.26)$ & $22.81 \%(13 / 57)$ \\
\hline$X^{2}$ & & & & & & 5.600 \\
\hline$P$ & & & & & & 0.018 \\
\hline
\end{tabular}

management so that nurses can quickly adapt to work. In this study, the total satisfaction after intervention was remarkably higher in the EG than in the CG $(P<0.05)$, suggesting that the combined intervention can help patients improve nursing satisfaction while eliminating their adverse emotions so that they can better cooperate with the treatment.

\section{Conclusion}

In conclusion, high-quality nursing intervention based on humanistic care combined with the project teaching method for AL patients undergoing chemotherapy can effectively relieve negative emotions and promote the development of positive coping styles, with high nursing satisfaction, which is worth applying and promoting. However, this study also has several limitations, such as the small sample size and lack of long-term follow-up observation of patients. Thus, we should expand the sample size with longer follow-up time to make the research results more objective.

\section{Data Availability}

The datasets used and/or analyzed during the current study are available upon request to the author.

\section{Conflicts of Interest}

The author declares that there are no conflicts of interest.

\section{References}

[1] M. L. Goh, E. N. K. Ang, Y.-H. Chan, H.-G. He, and K. Vehviläinen-Julkunen, "Patient satisfaction is linked to nursing workload in a Singapore hospital," Clinical Nursing Research, vol. 27, no. 6, pp. 692-713, 2018.
[2] S. A. N. G. H. Y. U. K. Park, M. E. E. J. E. O. N. G. Lee, and C. H. A. N.-J. E. O. U. N. G. Park, "Bone marrow recovery of hematopoietic stem cells and microenvironment after chemotherapy in childhood acute lymphoblastic leukemia: consecutive observations according to chemotherapy schedule[J]," Pediatric Hematology \& Oncology, vol. 36, no. 1/4, pp. 222-235, 2019.

[3] A. Samimi, M. Ghanavat, S. Shahrabi, S. Azizidoost, and N. Saki, "Role of bone marrow adipocytes in leukemia and chemotherapy challenges," Cellular and Molecular Life Sciences, vol. 76, no. 13, pp. 2489-2497, 2019.

[4] L. I. N. Fu, J. I. A. L. E. I. Qi, and X. I. A. N. G. Gao, "High expression of miR-338 is associated with poor prognosis in acute myeloid leukemia undergoing chemotherapy[J]," Journal of Cellular Physiology, vol. 234, no. 11 Pt 2, pp. 20704-20712, 2019.

[5] C. Duffy, L. Hall, J. Godown, T. Koyama, and S. C. Borinstein, "Steroid-induced b during induction chemotherapy in children and young adults diagnosed with acute lymphoblastic leukemia and lymphoblastic lymphoma," Journal of Pediatric Hematology, vol. 41, no. 7, pp. 537-541, 2019.

[6] K. E. L. I. N. M. Brace, W. E. I. W. E. I. Lee, and P. E. T. E. R. D. Cole, "Childhood leukemia survivors exhibit deficiencies in sensory and cognitive processes, as reflected by event-related brain potentials after completion of curative chemotherapy: a preliminary investigation[J]," Journal of Clinical and Experimental Neuropsychology, vol. 41, no. 7/8, pp. 814-831, 2019.

[7] N. Jain and S. O'Brien, "The spclleukemia," The Cancer Journal, vol. 25, no. 6, pp. 374-377, 2019.

[8] J. E. Cortes, R. J. Ryan, and M. Chiarella, "Pooled clinical safety analysis of CPX-351 versus conventional chemotherapy in patients with newly diagnosed or relapsed/refractory acute myeloid leukemia," Clinical Lymphoma, Myeloma \& Leukemia, vol. 19, no. Suppl.1, pp. S214-S215, 2019.

[9] S. L. Werth and R. Justice, "Prevalence of moisture-associated skin damage in an acute care setting," The Journal of Wound, 
Ostomy and Continence Nursing, vol. 46, no. 1, pp. 51-54, 2019.

[10] C. Leung, J. Leung, S. Leung, and W. Karnilowicz, "Pilot evaluation of the Whole Inclusive School Empowerment (WISE) project in kindergartens in Hong Kong: a mixed method approach," Psychology in the Schools, vol. 56, no. 1, pp. $42-55,2019$.

[11] P. Lebreton and T. Leguay, "First association between a Bispecific T-cell engager (BiTE) and an intensive chemotherapy in an adult population with refractory cytological B-cell acute lymphoblastic leukemia: a retrospective study," Clinical Lymphoma, Myeloma \& Leukemia, vol. 19, no. Suppl.1, pp. S179-S180, 2019.

[12] C. K. Meenan, J. A. Kelly, L. Wang, A. K. Ritchey, and S. H. Maurer, "Obesity in pediatric patients with acute lymphoblastic leukemia increases the risk of adverse events during pre-maintenance chemotherapy," Pediatric Blood and Cancer, vol. 66, no. 2, p. e27515, 2019.

[13] I. Tsutsumi, S. Kunisawa, C. Yoshida et al., "Impact of oral voriconazole during chemotherapy for acute myeloid leukemia and myelodysplastic syndrome: a Japanese nationwide retrospective cohort study," International Journal of Clinical Oncology, vol. 24, no. 11, pp. 1449-1458, 2019.

[14] A. Militaru, S. Zus, A. M. Cimpean et al., "Early dc in patients undergoing chemotherapy for acute lymphoblastic leukemia," Anticancer Research, vol. 39, no. 6, pp. 3255-3264, 2019.

[15] A. M. Brunner, D. S. Neuberg, S. A. Wander et al., "Isocitrate dehydrogenase 1 and 2 mutations, 2-hydroxyglutarate levels, and response to standard chemotherapy for patients with newly diagnosed acute myeloid leukemia," Cancer, vol. 125, no. 4, pp. 541-549, 2019.

[16] J. Dotson, A. Elhamdani, E. Petryna, M. O. Jamil, and M. Alsharedi, "Neutropenic enterocolitis in patients with FLT3 mutated acute myeloid leukemia undergoing induction chemotherapy with midostaurin," International Journal of Hematology, vol. 109, no. 3, pp. 351-355, 2019.

[17] S. Fellah, Y. T. Cheung, M. A. Scoggins et al., "Brain activity associated with attention deficits following chemotherapy for childhood acute lymphoblastic leukemia," Journal of the National Cancer InstituteJournal of the National Cancer Institute, vol. 111, no. 2, pp. 201-209, 2019.

[18] S. I. N. A. Zabihi, R. A. S. H. I. D. I. Kahag Mahdi, and H. A. M. I. D. R. E. Z. A. Maghsoudlou, "Multi-objective teaching-learning-based meta-heuristic algorithms to solve multi-skilled project scheduling problem[J]," Computers \& Industrial Engineering, vol. 136, pp. 195-211, 2019.

[19] M. A. Davidson, C. M. Dewey, and A. E. Fleming, "Teaching communication in a statistical collaboration course: a feasible, project-based, multimodal curriculum," The American Statistician, vol. 73, no. 1, pp. 61-69, 2019.

[20] V. Cantoni, P. Dondi, L. Lombardi, and A. Setti, "Teaching computer graphics through a digital humanities project," IEEE Computer Graphics and Applications, vol. 39, no. 2, pp. 89-94, 2019.

[21] S. A. R. A. Martínez-Cardama and M. E. R. C. E. D. E. S Caridad-Sebastián, "Social media and new visual literacies: proposal based on an innovative teaching project[J]," Education for Information, vol. 35, no. 3, pp. 337-352, 2019.

[22] J. I. N. G. X. I. A. O. Zhang, H. A. I. Y. A. N. Xie, and H. U. I Li, "Project based learning with implementation planning for student engagement in BIM classes[J]," International Journal of Engineering Education, vol. 35, no. 1B, pp. 310-322, 2019.
[23] H. Lee and J.-e. Yang, "Science teachers taking their first steps toward teaching $\mathrm{s}$ issues through collaborative action research," Research in Science Education, vol. 49, no. 1, pp. 51-71, 2019.

[24] S. Frohnapfel and R. Frohnapfel, "Enhancing students' experimental planning competences by introducing and evaluating the IS4O teaching approach," Journal of Biological Education, vol. 53, no. 5, pp. 541-547, 2019.

[25] W. Yang, N. Yuan, W. Chinthammit, and B. Kang, "A distributed case- and project-based learning to design $3 \mathrm{D}$ lab on electronic engineering education," Computer Applications in Engineering Education, vol. 27, no. 2, pp. 430-451, 2019. 